\title{
Wide-field variability survey of the globular cluster NGC 4833
}

\author{
Grzegorz Kopacki \\ Instytut Astronomiczny, Uniwersytet Wrocławski, \\ Kopernika 11, 51-622 Wrocław, Poland \\ email: kopacki@astro.uni.wroc.pl
}

\begin{abstract}
We present preliminary results of the variability survey in the field of the globular cluster NGC 4833. We observed all 34 variable stars known in the cluster. In addition, we have found two new SX Phoenicis stars, one new RR Lyrae star, twelve new eclipsing systems mostly of the $\mathrm{W}$ Ursae Majoris type, nine new variable red giants, and ten new field-stars showing irregular variations. Properties of RR Lyrae stars indicate that NGC 4833 is an Oosterhoff's type II globular cluster.
\end{abstract}

Keywords. Galaxy: globular clusters: individual: NGC 4833, stars: Population II, stars: variables: RR Lyrae, stars: variables: SX Phoenicis

\section{Introduction}

Continuing our ongoing project aimed at the search and analysis of pulsating stars in globular clusters (see Kopacki 2013) we present preliminary results for NGC 4833. We used image subtraction method (ISM, Alard \& Lupton 1998) which works well in crowded stellar fields like a cluster core and thus enables detection of many variable stars, such as RR Lyrae and SX Phoenicis stars.

NGC 4833 is the southern globular cluster of intermediate metallicity $([\mathrm{Fe} / \mathrm{H}]=$ -1.85). The most recent version of the Catalogue of Variable Stars in Globular Clusters (CVSGC, Clement et al. 2001) listed 34 objects in the field of this cluster including six SX Phoenicis stars and 20 RR Lyrae stars.

\section{Observations and results}

We used CCD observations obtained during a one-month observing run in Feb/Apr, 2008 using 40-inch telescope at Siding Spring Observatory, Australia. They consisted of $740 \mathrm{~V}$-filter and $220 I_{\mathrm{C}}$-filter CCD frames.

We confirmed all variable stars found recently in the cluster core by Darragh \& Murphy (2012). In addition, we have detected two new SX Phoenicis stars, one new RR Lyrae star, twelve new eclipsing systems mostly of the W Ursae Majoris type, nine new variable red giants at the tip of the red giant branch, and ten field-stars showing irregular variations. Equatorial coordinates of periodic variable stars we observed, together with derived periods, are given in Table 1 . New variable stars are indicated with designations starting with letter ' $n$ '.

The mean period of RRab stars in NGC 4833 is equal to $\left\langle P_{\mathrm{ab}}\right\rangle=0.701 \mathrm{~d}$, and the relative percentage of RRc stars amounts to $N_{\mathrm{c}} /\left(N_{\mathrm{ab}}+N_{\mathrm{c}}\right)=48 \%$. With these values we find that NGC 4833 belongs to the Oosterhoff's II group of globular clusters. 
Table 1. Equatorial coordinates and periods of periodic stars in NGC 4833.

\begin{tabular}{|c|c|c|c|c|}
\hline Var & קDe & ${ }^{\mathrm{h}}$ & ${ }^{\circ}$ & $P[\mathrm{~d}]$ \\
\hline $\begin{array}{l}\mathrm{v} 27 \\
\mathrm{v} 29 \\
\mathrm{v} 30 \\
\mathrm{v} 31 \\
\mathrm{v} 32 \\
\mathrm{v} 33 \\
\mathrm{n} 35 \\
\mathrm{n} 36\end{array}$ & $\begin{array}{l}\text { SXPhe } \\
\text { SXPhe } \\
\text { SXPhe } \\
\text { SXPhe } \\
\text { SXPhe } \\
\text { SXPhe } \\
\text { SXPhe } \\
\text { SXPhe }\end{array}$ & $\begin{array}{lll}12 & 59 & 13.73 \\
12 & 59 & 35.31 \\
12 & 59 & 42.56 \\
12 & 59 & 47.98 \\
12 & 59 & 55.05 \\
12 & 59 & 57.64 \\
12 & 58 & 59.01 \\
12 & 59 & 32.64\end{array}$ & \begin{tabular}{|llll}
-70 & 52 & 09.5 \\
-70 & 52 & 40.9 \\
-70 & 53 & 04.0 \\
-70 & 52 & 51.5 \\
-70 & 52 & 24.2 \\
-70 & 54 & 29.5 \\
-70 & 52 & 05.1 \\
-70 & 53 & 15.9
\end{tabular} & 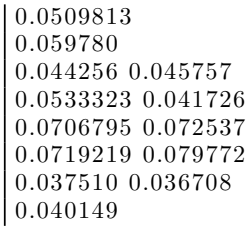 \\
\hline $\begin{array}{l}\mathrm{v} 01 \\
\mathrm{v} 03 \\
\mathrm{v} 04 \\
\mathrm{v} 05 \\
\mathrm{v} 06 \\
\mathrm{v} 07 \\
\mathrm{v} 12 \\
\mathrm{v} 13 \\
\mathrm{v} 14 \\
\mathrm{v} 15 \\
\mathrm{v} 17 \\
\mathrm{v} 18 \\
\mathrm{v} 19 \\
\mathrm{v} 20 \\
\mathrm{v} 21 \\
\mathrm{v} 22 \\
\mathrm{v} 23 \\
\mathrm{v} 24 \\
\mathrm{v} 26 \\
\mathrm{v} 28 \\
\mathrm{n} 37\end{array}$ & $\begin{array}{l}\text { RRLyr } \\
\text { RRLyr } \\
\text { RRLyr } \\
\text { RRLyr } \\
\text { RRLyr } \\
\text { RRLyr } \\
\text { RRLyr } \\
\text { RRLyr } \\
\text { RRLyr } \\
\text { RRLyr } \\
\text { RRLyr } \\
\text { RRLyr } \\
\text { RRLyr } \\
\text { RRLyr } \\
\text { RRLyr } \\
\text { RRLyr } \\
\text { RRLyr } \\
\text { RRLyr } \\
\text { RRLyr } \\
\text { RRLyr } \\
\text { RRLyr }\end{array}$ & $\begin{array}{lll}12 & 58 & 36.53 \\
12 & 59 & 33.73 \\
12 & 59 & 33.76 \\
13 & 00 & 01.28 \\
12 & 59 & 56.18 \\
12 & 59 & 48.80 \\
12 & 59 & 37.91 \\
13 & 00 & 29.86 \\
12 & 59 & 31.04 \\
12 & 59 & 20.15 \\
12 & 59 & 43.98 \\
12 & 59 & 28.26 \\
12 & 59 & 06.02 \\
12 & 59 & 08.21 \\
12 & 59 & 50.94 \\
12 & 59 & 45.10 \\
12 & 59 & 44.74 \\
12 & 59 & 36.68 \\
12 & 59 & 02.74 \\
12 & 59 & 21.22 \\
12 & 59 & 43.16\end{array}$ & \begin{tabular}{|llll}
-70 & 44 & 48.1 \\
-70 & 52 & 13.2 \\
-70 & 51 & 57.8 \\
-70 & 53 & 17.3 \\
-70 & 50 & 10.2 \\
-70 & 52 & 21.1 \\
-70 & 52 & 14.7 \\
-70 & 52 & 55.9 \\
-70 & 53 & 06.9 \\
-70 & 53 & 25.0 \\
-70 & 54 & 24.9 \\
-70 & 54 & 25.5 \\
-70 & 53 & 29.7 \\
-70 & 52 & 23.5 \\
-70 & 50 & 36.5 \\
-70 & 53 & 55.5 \\
-70 & 51 & 27.0 \\
-70 & 52 & 58.7 \\
-70 & 52 & 51.9 \\
-70 & 53 & 26.4 \\
-70 & 53 & 36.5
\end{tabular} & \begin{tabular}{|l}
0.750082 \\
0.74453 \\
0.65577 \\
0.629424 \\
0.65400 \\
0.66888 \\
0.58980 \\
0.36788 \\
0.40842 \\
0.66745 \\
0.390263 \\
0.42559 \\
0.370658 \\
0.29970 .3012 \\
0.39878 \\
0.85095 \\
0.406503 \\
0.62612 \\
0.31788 \\
0.87401 \\
0.30215
\end{tabular} \\
\hline $\begin{array}{l}\text { v25 } \\
\text { v34 } \\
\text { n38 } \\
\text { n39 } \\
\text { n40 } \\
\text { n41 } \\
\text { n42 } \\
\text { n43 } \\
\text { n44 } \\
\text { n45 } \\
\text { n46 } \\
\text { n47 } \\
\text { n48 } \\
\text { n49 }\end{array}$ & \begin{tabular}{|l|}
$E c l$ \\
$E c l$ \\
$E c l$ \\
$E c l$ \\
$E c l$ \\
$E c l$ \\
$E c l$ \\
$E c l$ \\
$E c l$ \\
$E c l$ \\
$E c l$ \\
$E c l$ \\
$E c l$ \\
Ecl
\end{tabular} & $\begin{array}{lll}12 & 58 & 55.37 \\
13 & 00 & 25.17 \\
13 & 00 & 11.88 \\
13 & 01 & 33.10 \\
13 & 01 & 49.41 \\
13 & 01 & 29.29 \\
12 & 57 & 17.71 \\
13 & 00 & 22.56 \\
12 & 59 & 52.75 \\
13 & 00 & 43.49 \\
13 & 00 & 24.25 \\
12 & 59 & 37.69 \\
13 & 00 & 45.49 \\
13 & 00 & 55.03\end{array}$ & \begin{tabular}{|lll}
-70 & 51 & 45.9 \\
-70 & 49 & 16.4 \\
-70 & 51 & 26.4 \\
-70 & 49 & 22.6 \\
-70 & 51 & 42.1 \\
-70 & 44 & 58.0 \\
-70 & 47 & 51.0 \\
-70 & 55 & 25.8 \\
-70 & 44 & 32.7 \\
-70 & 47 & 26.9 \\
-70 & 56 & 01.4 \\
-70 & 51 & 28.4 \\
-70 & 47 & 38.2 \\
-70 & 54 & 15.9
\end{tabular} & \begin{tabular}{|l}
0.72310 \\
0.36290 \\
0.257129 \\
0.28005 \\
0.282581 \\
0.29917 \\
0.3137 \\
0.3175 \\
0.3362 \\
0.362332 \\
0.38071 \\
0.48875 \\
0.49226 \\
1.1235
\end{tabular} \\
\hline $\begin{array}{l}\mathrm{n} 59 \\
\mathrm{n} 60 \\
\mathrm{n} 61\end{array}$ & $\begin{array}{l}\text { Ukn } \\
\text { Ukn } \\
\text { Ukn }\end{array}$ & $\begin{array}{lll}13 & 00 & 27.52 \\
12 & 59 & 14.62 \\
13 & 00 & 20.85\end{array}$ & $\mid \begin{array}{llll}-70 & 54 & 01.6 \\
-70 & 50 & 37.8 \\
-70 & 49 & 56.4\end{array}$ & \begin{tabular}{|l}
2.889 \\
4.390 \\
6.300
\end{tabular} \\
\hline
\end{tabular}

Almost all observed SX Phoenicis stars show multiperiodic light changes (see Table 1) with one star, v31, exhibiting oscillation in two first radial modes. Moreover, we found in the RRc star v20 two closely-spaced frequencies.

\section{Acknowledgement}

This work was supported by the NCN grant 2011/03/B/ST9/02667.

\section{References}

Alard, C. \& Lupton, R. 1998, ApJ, 503, 325

Clement, C., Muzzin, A., Dufton, Q., et al. 2001, AJ, 122, 2587

Darragh, A. \& Murphy, B. 2012, Journal of the Southeastern Association for Research in Astronomy, 6, 72 .

Kopacki, G. 2013, AcA, 63, 91 